\title{
WAP-based Comprehensive Inquiry System on Immune Systems Index Xiaofei Liu
}

\author{
Bohai University, Jinzhou, P.R. China
}

3079134095@qq.com

Keywords: Immune system; WAP technology; index monitoring; indexes query; immune globulin

\begin{abstract}
With the growing popularity and in-depth of scientific monitoring work, players' body shape has become a basic everyday work for the current athletic teams, in which the monitoring of immune system index is an important part of daily work, so the comprehensive inquire system on the immune system index based on WAP technology researched in this paper has certain practical significance. Firstly, this paper studies the WAP technical framework, including its development and basic principles; secondly, studies the immune system index, including $\mathrm{T}$ lymphocytes, white blood cells, immune globulin and so on; finally studies the comprehensive inquire system on the immune system index, including the query technique used in the system and so on. WAP-based the comprehensive inquire system on the immune system index designed in this paper promotes to carry out the monitoring work and play a protective role for athletes.
\end{abstract}

\section{Introduction}

With the growing popularity and in-depth of scientific monitoring work for athletes' body, athletes' body shape and performance testing has become two basic routine for current athletic teams at all levels, various athletic teams and research institutions have accumulated a lot of athlete and performance testing data. The relationship between exercise and immunity earliest started in the 19th century, in recent years, with the rapid development of modern immunology and molecular biology techniques, the relationship between exercise and immunity increasing obtained attention. Athletes' immunity is closely related to the physical condition, in practice, some athletes body's defenses are weakened after exercise or competition, often infected with diseases and even affect athletic ability, decreased motion immune function is a long-term thorny problem for athletes and coaches, during high intensity exercise or competition athletes can use the querying of immune system index to early detect changes in immune function, and take measures, so that preventive measures are very important [1]. Therefore, WAP-based comprehensive inquiry system on immune system index designed in this paper has some practical significance.

WAP technology is a mobile technology, mobile technology has many definitions, in which A definition refers to a instrument which in a different form to access, generate, modify, organize and store information in a place, it can be said that mobile technology is a general designation, at any time and any place people can exchange information technology and related equipment [2]. Mobile technology includes four techniques, which are communication or broadcasting based on radio; mobile voice service, SMS (Short Message Service), WAP (Wireless Application Protocol), GPRS (General Packet Radio Service), UMTS (3G i.e. ) based on cellular phones; laptops, GPS (global Positioning system) based on mobile devices; Wi-Fi-based networks. This paper mainly used WAP technology.

\section{WAP Technical Framework}

Since the mid-1990s, mobile communications technology and Internet technology has obtained rapid development and greatly changed people's lives, becoming two hot in the IT development. The development of mobile communication technology can make people contact anytime, anywhere, Internet used a wired connection, but mobile communication used wireless technology, if people want to get information on the Internet they can only use installed computer in fixed location, Internet provides mainly digitization information, but wireless communication technology mainly 
provides voice services, cannot meet the need for people to access and interact with information on the moving. In mobile telephony, the world's mobile users has reached 2.1 billion, with the rapid expansion of the mobile phone market and fierce competition, improving the technical content and allowing users to obtain information have become an inevitable choice for mobile handset manufacturers. The combination of the two technologies to meet the people to obtain information on the moving becomes a new technology that is WAP technology [3], WAP technology is mainly used in information services, e-commerce, and entertainment services and so on.

WAP is a global unified open protocol standard, provided Internet content and advanced value-added services for mobile terminals. Its main designed objective is to provide an optimized open standard for mobile devices to access the Internet or an intranet. WAP technology makes people get rid of the shackles of time and space and the network cable, WAP network architecture consists of three parts: WAP gateway, WAP phones and WAP servers. WAP gateway plays a role of translation for protocol, is a bridge connected the GPRS network with Internet; WAP server stores information to provide access, query, browse, etc for WAP mobile phone users. When the user input URL to be accessed WAP server from the WAP phone, signal through a wireless network to send a request to WAP gateway in form of the WAP protocol, and then through the "translation" of gateway, then in form of HTTP protocol to interact with the WAP content server, finally WAP gateway will return the compressed and processed contents by the way of binary stream the customer's WAP phone screen [4]. The basic framework is shown in Fig. 1.

\begin{tabular}{|c|c|c|c|c|}
\hline \multirow{2}{*}{$\begin{array}{l}\text { Mobile terminal } \\
\text { (WAP phone) }\end{array}$} & WAP Protocol & \multirow{2}{*}{$\begin{array}{l}\text { WAP Gateway } \\
\text { (GPRS-Internet) }\end{array}$} & HTTP Protocol & WAP Server \\
\hline & Encoding request & & URL request & (Store info) \\
\hline WAP Users & Decoding response & $\begin{array}{l}\text { Encoding and } \\
\text { decoding }\end{array}$ & File resnonse & CGI RAM \\
\hline
\end{tabular}

Fig. 1. WAP basic framework diagram

\section{Immune System Index}

The immune system is most important defend system that the body against pathogens, consisted of the immune organs, immune cells and immune molecules. The immune system is divided into innate immunity and adaptive immunity, its primary function is to fight infectious, potential and hazardous germs, due to the immune system contains a number of different cells, so when they work need the transmission of information between them. A lot of information is delivered by cytokines and chemical factors. Due to the response results produced by high intensity excise cause a decline in the immune index, including the reduction in the number of lymphocytes, inhibiting of function; NK cells, phagocyte cell function decline. At the same time, after high-intensity excise the number of neutrophils will increase in the blood, serum interleukin also significantly increased, the changes of the immune system caused by exercising is short, only last 3-72 hours, so changes of athletes' immune parameters are very similar to non-athletes [5].

Immunity of athletes is closely related with the physical condition, but due to decreased immune function has seriously affected the athletic ability, immunological tests can make athletes understand the functional state of the immune system, and targeted to take measures to avoid reduced immune function as far as possible caused by fatigue or psychological stress, reasonably arrange exercise and adjust the immune system function have a positive effect. When monitor the immune system for the athletes, now usually used immunoglobulin and lymphocyte subsets and other indexes. Similarly, to understand the relationship between these immune parameters has great positive effect to assess immunity and effective exercise arrangements [6]. So the comprehensive inquiry the immune system index is important, the specific querying index of immune system is shown in Table 1. 
Table 1. Querying index of immune system

\begin{tabular}{c|c|c|c|c}
\hline No & ClassID & Index name & Abbreviation & Measure unit \\
\hline 1 & 030101 & T lymphocytes (CD3) & TL & $\%$ \\
\hline 2 & 030102 & Helper T-cell (CD4) & TH & $\%$ \\
\hline 3 & 030103 & Suppressor T-cell (CD8) & TS & $\%$ \\
\hline 4 & 030105 & B lymphocytes (CD19) & BL & $\%$ \\
\hline 5 & 030106 & Natural Killer Cell & NK Cell & $\%$ \\
\hline 6 & 030201 & White Blood Cell Count (S, M, L) & WBC & $10 \mathrm{E} 9 / \mathrm{L}$ \\
\hline 7 & 030301 & Immunoglobulin (IgA, IgB...IgM) & Ig & $\mathrm{g} / \mathrm{L}$ \\
\hline 8 & 030401 & Complement (C1,C2....C9) & C & $\mathrm{g} / \mathrm{L}$ \\
\hline
\end{tabular}

\section{Comprehensive Inquiry System Design}

The comprehensive inquire system on the immune system index designed in this paper provides a platform for end users querying the index, the user can use the index full name, acronym, query keywords and others to query, the returned result is the user's specific number about immune system index, and then by the index value to determine the user's immune system health. It mainly included user login module, the user registration module and index comprehensive inquiry module. User login module is mainly used for user login system, users input user name, password, etc. and to enter the system, when login the system will connect to the database to determine whether the information entered by the user is correct. User Registration module is mainly used to register user information to log on the system querying index [7]. index comprehensive inquiry module is a key part of WAP-based technology comprehensive inquiry system on the immune system index designed in this paper, is mainly used for querying the user's immune system index. Its system flow diagram is shown in Fig. 2.
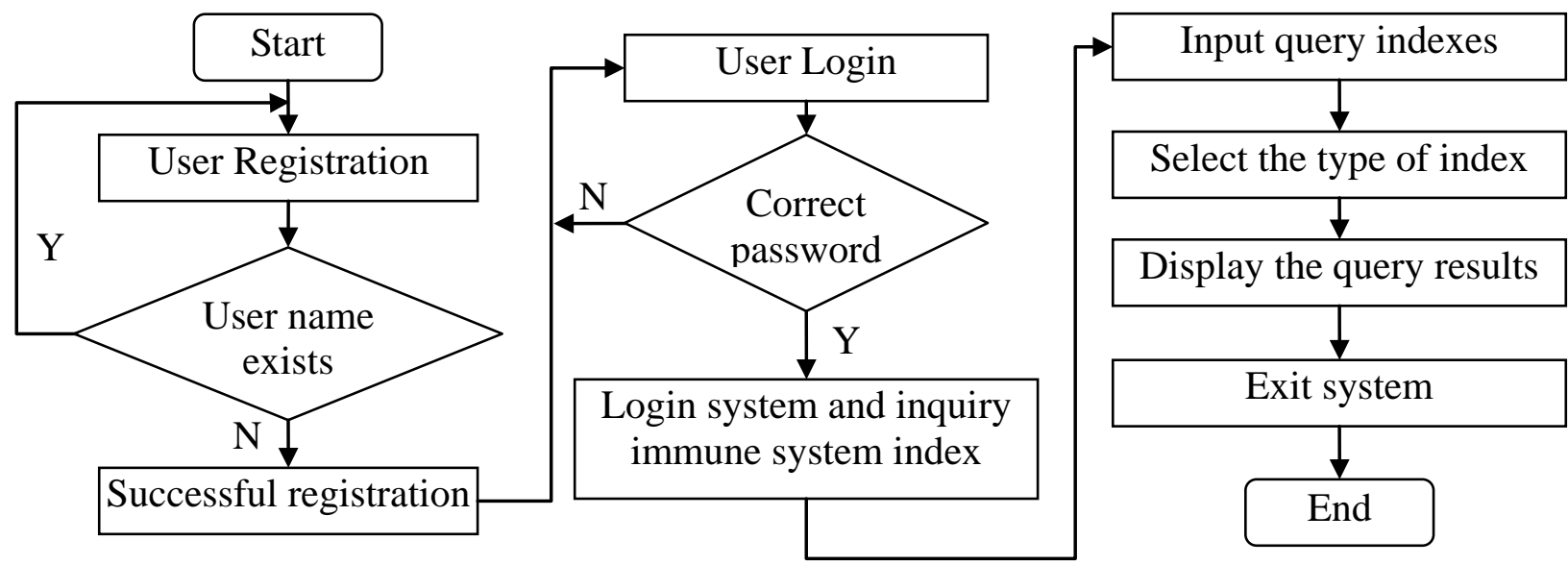

Fig. 2. Immune system index comprehensive inquiry system flow chart

in which, the technology used in index comprehensive inquiry is database query technology, currently the most widely used database mainly includes two categories, namely, distributed databases and parallel databases, in the operating of database, the query is the most common operations for database, it is also the only way that the user to manipulate and maintain data in the database, the direct feeling of database performance is whether the query processing of database management system is efficient, fast, the efficient of query processing determines the performance of the database management system, even determines the performance of the system, so in order to improve query speed, system speed and performance this paper used indexing technology. The nature of indexing techniques is to add restrictions to reduce the amount of data scanning, so indexing techniques can reduce query time, and then optimize the query results. Currently the 
indexing technology used query optimization is mainly divided into three categories: hashing technique, tree-based indexes and bitmap indexes, in which due to the index table occupied larger memory space, so it is generally adopted hashing indexing in order to reduce system overhead [8]. This paper establishes an indexing based on immune system index in order to improve the query performance of the immune system index comprehensive inquiry system and provide users with better, faster and more convenient querying platform. So the contents of this paper hope to make some contribution for athletes' healthy immune system, meanwhile lay the foundation for scientific exercising monitoring.

\section{Conclusion}

At present mobile phone users has more than 360 million, it is several times as the fixed network users. Through the Wireless Application Protocol (WAP), mobile phone users can also access the Internet for obtaining information and network resources, compared to using PC connected to the Internet, the biggest feature of using wireless is the flexibility of mobile handsets, as long as in the GSM network coverage area, you can mobile access Internet via WAP [9]. With the growing popularity and in-depth of scientific monitoring work for athletes' body, athletes' body shape and performance testing has become two basic routine, athletes' immunity is closely related to the physical condition, in practice, some athletes body's defenses are weakened after exercise or competition, and even affect athletic ability, therefore, by querying the immune system index early finding the changes in immune function is necessary. This paper discusses the relevant technology and implementation principle of the immune system index comprehensive inquiry system on basis of WAP technology, from the principle analysing the immune system index query system can be realized, as WAP wireless technology continues to mature, the design of WAP-based wireless Internet application will be extended to more areas [10].

\section{References}

[1] Y. Fang, X. Y. Pei, W. Z. Li, B. X. Fan, J. Z. Li, X. L. Jin, "Effect of High Intensity Training on the Speed Skaters' Immunity Indexes," China Winner Sports, vol. 32, no. 2, pp. 58-60, 2010.

[2] J. L. Chang, "Discussion and Research on Mobile Technology," Value Engineering, vol. 30, no. 30, pp. 140-141, 2011.

[3] Read K, Maurer F. "Developing Mobile Wireless Application," IEEE Internet Computing, vol. 4, no. 1, pp. 81-86, 2003.

[4] F. Yang, "The Research of Traffic Accident Intelligent Transation System Based on WAP," Master's Degree of Changsha University of Technology, 2007.

[5] R. C. Li, C. Y. Wang, S. C. Li, "Effect of Training Intensity on the Athlete's Immunity System Function," China Winner Sports, vol. 26, no.5, pp. 110, 2004.

[6] P. W. Feng, S. L. Feng, P. Hong, R. L. Wang, "Correlative Analysis on Immune SystemFunction of Our Elite Swimmers," China Sport Science, vol. 25, no. 4, pp. 32-34, 2005.

[7] L. X. Jiao, J. L. Li, "Study onWireless Query Systems Development Based on WAP," Science Technology and Engineering, vol. 7, no. 10, pp. 2374-2375, 2007.

[8] S. Li, "Database Query Optimization Research," Computer Knowledge and Technology, vol. 6, no. 21, pp. 6103-6104, 2010.

[9] Y. C. Wu, K. Q. Tan, Q. Ou'yang, Z. F. Han, "Bus Schedules Query System Based on WAP," Control \& Automation, vol. 24, no. 5, pp. 108-109, 2007.

[10] B. W. Huang, B. W. Huang, "Design and Implementation of WAP-based Wireless Internet Bus Query System," Computer Era, vol. 28, no. 5, pp. 33-34, 2010. 